\title{
Quantitative and bi-modal (optical-MRI) imaging of the vulnerable atherosclerotic plaque in the mouse with specific functional markers
}

\author{
Emmanuelle Canet-Soulas ${ }^{1}$ \\ University of Lyon 1, CREATIS-LRMN, UMR CNRS 5220, U630 INSERM \\ La Doua, Bâtiment CPE, 43 Boulevard du 11 Novembre 1918 \\ Lyon, France \\ Email:emmanuelle.canet@univ-lyon1.fr
}

Didier Letourneur

University of Paris 7, U698 INSERM,

Paris, France

Email: didier.letourneur@bichat.inserm.fr

\begin{abstract}
It is well accepted that major acute events in cardiovascular diseases are the consequences of the rupture of a vulnerable atherosclerotic plaque. The main issue for medical imaging is thus to diagnose vulnerable lesions before rupture and sudden vessel occlusion. The pathogenesis of atherosclerosis contains an important inflammatory component, which has been recognized as a key process in the malignant evolution of the disease. Magnetic Resonance Imaging (MRI) has recently evolved from a powerful non invasive morphological diagnostic technique to a more functional one, and is rapidly moving to molecular imaging thanks to the conception of new MRI contrast agents. Progress in molecular MRI of atherosclerosis is performed using animal models, where it is necessary to develop adequate methodology to enable in-vivo plaque imaging both in rabbits and mice. The next step is to synthesize an MR contrast agent targeted to inflammation in the plaque. For this, various targeting strategies are under evaluation. In our example, a gadolinium contrast agent was designed to target adhesion molecules highly expressed in active atherosclerotic plaques. Then, the arterial wall contrast enhancement was evaluated in vivo in ApoE-/- mice after the intravenous injection of the new macromolecular gadolinium agent, functionalized to target inflammation. To enable dual detection by MRI and fluorescence imaging, a fluorochrome was grafted on the gadolinium platform.
\end{abstract}

Frontiers in Imaging Science: High Performance Nuclear Medicine Imagers for Vascular Disease Imaging (Brain and Heart) Istituto Superiore di Sanita', Rome, Italy 13-14 November, 2006

\footnotetext{
1 Speaker
} 


\section{Introduction}

It is well accepted that major acute events in cardiovascular diseases are the consequences of the rupture of a vulnerable atherosclerotic plaque. The main issue for diagnostic using imaging modalities is thus to diagnose vulnerable lesions before their rupture and its dramatic cascade of events, i.e. thrombus formation and sudden vessel occlusion. The pathogenesis of atherosclerosis contains an important inflammatory component, which has been recognized as a key process in the malignant evolution of the disease. It can be described by a series of specific cellular and molecular responses \{Libby, 2002\}. Functional parameters such as vessel wall transport between blood and artery wall also play an important role in early stages of atherosclerosis and appear to change progressively with the evolution of the disease $\{\mathrm{Wu}, 2006$; McEver, 1997\}.

Magnetic Resonance Imaging has recently evolved from a powerful non invasive morphological diagnostic technique to a more functional one, and is rapidly moving to molecular imaging thanks to the conception of new MRI contrast agents \{Winter, 2003; Jaffer, 2006\}. The first clinical application of non specific MR contrast agents for plaque imaging has been for the delineation of the fibrous cap that is covering a lipid rich core in an advanced and dangerous atherosclerotic lesion \{Stary, 1995; Yuan, 2002; Wasserman, 2002\}. As a thin fibrous cap is an indicator of a vulnerable plaque, paramagnetic gadolinium MR contrast agent, diffusing from the blood stream into the fibrous cap, were found very helpful to examine its thickness. Iron oxide nanoparticles, the second family of MRI contrast agent, are also seen as very promising as they are taken up by phagocytic cells, which are very important actors in inflamed and active atherosclerotic plaques \{Nighoghossian, 2005; Ruehm, 2001; Kooi, 2003\}.

\section{MRI of atherosclerosis in animal models}

Advances in the understanding of this complex multifactorial disease and the development of new drugs and markers of atherosclerosis are mostly performed using genetically selected (the Watanabe rabbit) or genetically modified (the Apolipoprotein knock-out or ApoE-/- mouse) animal models. The ApoE-/- mouse is well known to develop atherosclerotic lesions that have similar characteristics, evolution and distribution to those found in humans \{Coleman, 2006\}. The reference to evaluate plaque in these animals is still histopathology (Figure 1). Thus, to explore in-vivo by MRI the functional state of the atherosclerosis plaque and its risk, it is necessary to develop adequate methodology to enable plaque imaging in these animal models. The first prerequisite is to achieve the requested spatial resolution (Figure 1) in the minimum acquisition time. For mice imaging, the preferred spatial resolution is an isotropic voxel with dimensions of less than $100 \mu \mathrm{m}$ (Figures 1 and 2). 


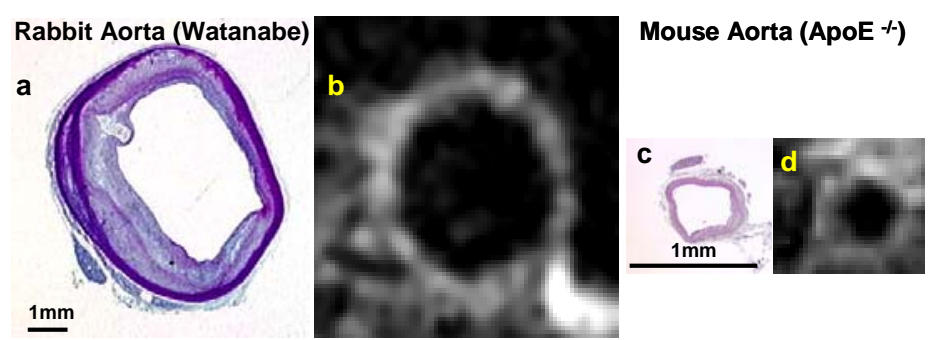

Figure 1: Histopathology (Hematoxylin-Eosin staining) $(a, c)$ and in vivo MRI of atherosclerosis in animal models $(b, d)$, highlighting high spatial resolution prerequisite for MRI: pixel size of 200-300 $\mu \mathrm{m}$ for rabbit aorta (lumen diameter $=5 \mathrm{~mm}$, vessel wall thickness $=$ 0.4-1.3 mm) (b) and pixel size of 70-90 $\mu \mathrm{m}$ for mouse aorta (lumen diameter $=0.5 \mathrm{~mm}$, vessel wall thickness $=40-200 \mu \mathrm{m})(\mathrm{d})$.

For the measurement of plaque volume, high spatial resolution could be achieved ex vivo after hours of MRI acquisition (Figure 2a). However, it is far more challenging to find the best compromise in vivo.

In this context, blood pool MRI contrast agents are very useful (Figure 2b) as they remain in the blood circulation for a long time and are more efficient on MR signal compared to non specific gadolinium agent. Briefly, paramagnetic gadolinium agents modify the contrast on proton MRI by their effects on the relaxation rate of protons in their neighbourhood, the socalled relaxivity. Thus, an efficient gadolinium agent is the one with the higher in vivo relaxivity, i.e. accelerating the proton relaxation nearby. For better evaluation of an MR contrast agent, the choice of the parameters of the MR sequence is essential: for paramagnetic gadolinium agents, highly T1-weighted sequences are requested to view their effect as a bright signal on MR images (Figure 2b).

In summary, for plaque imaging in the mouse aorta, one has to implement a robust high spatial resolution and high temporal resolution T1-weighted sequence and to inject intravenously a specific MR contrast agent to help characterization (Figure 2b) \{Chaabane, 2004; Chaabane, 2003; McFadden, 2004\}. 


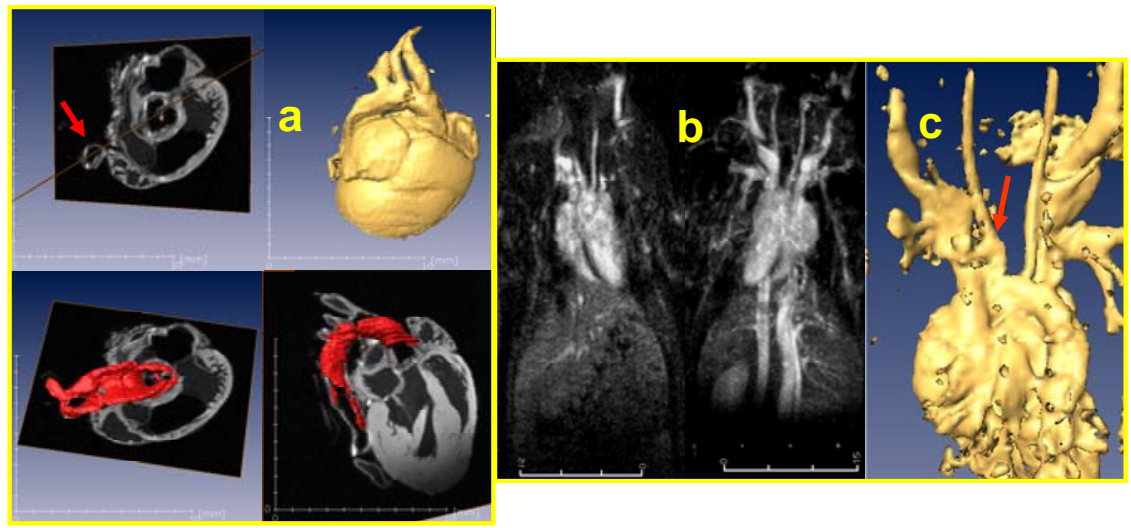

Figure 2: Ex vivo MRI characterization of atherosclerotic plaque volume (a, red segment, $\sim 6.3 \mathrm{~mm} 3$ ) with isotropic high spatial resolution 3D MRI (voxel size $=66 \times 66 \times 86 \mu \mathrm{m} 3$ ) in ApoE-/- mouse (a) and in vivo high temporal and high spatial resolution MR Angiography (voxel size $=211 \times 211 \times 234 \mu \mathrm{m} 3$, and acquisition time $=1 \mathrm{~min} 46 \mathrm{sec}$ ) with a blood pool MR contrast agent in the ApoE-/- mouse (b) \{Chaabane, 2000\}. The 3D reconstruction of the aortic arch and the carotids origins (c) shows the innominate trunk (arrow) which has been shown to develop vulnerable type lesions.

\section{Specific functional markers for dual optical-MRI detection}

To evaluate the risk associated with an atherosclerotic plaque, the major issue is to obtain a comprehensive view of ongoing cellular and molecular processes (Figure 3). It is envisioned that this could be achieved by molecular imaging techniques using dedicated markers, i.e. radioactive tracers, fluorescent probes, or specific magnetic resonance, $\mathrm{X}$ ray or ultrasound contrast agents. MRI contrast agents have very recently made a substantial progress in this field especially for cardiovascular applications, as evidenced by the number of original publications and reviews on the subject \{Weinreb, 2007; Sosnovik, 2007; Sosnovik, 2007; Wickline, 2006; Winter, 2003; Jaffer, 2006; Gupta, 2005; Corot, 2006; Aikawa, 2007; Amirbekian, 2006\}. Because of the complexity and interrelationship of ongoing cellular and molecular processes in atherosclerosis, the selection and the design of the best marker of plaque vulnerability is still a matter of debate (Figure 3). Should it be a ligand for a receptor expressed by activated cells, such as endothelial cells, macrophages, platelets, leucocytes and smooth muscle cells, or a marker for lipids or enzymes is an open question (Figure 3).

Thus, after an intravenous injection and an initial biodistribution in blood, the molecular imaging agent should be retained on the diseased vessel wall via a specific interaction and act differently compared to its non specific control agent (Figure 3). It has also to be detected by the imaging modality, in our case MRI. For that, the marker should bear both the specific chemical function to stick on the plaque, and the gadolinium or iron ions to modify the MRI contrast. As MRI has a very poor sensitivity, the agent has to be present at a detectable concentration, typically in the nano or even micromolar range. A high relaxivity contrast agent is generally preferred, but a fluorochrome can also be added to this complex structure to gain in sensitivity with detection by optical imaging (Figure 3). 


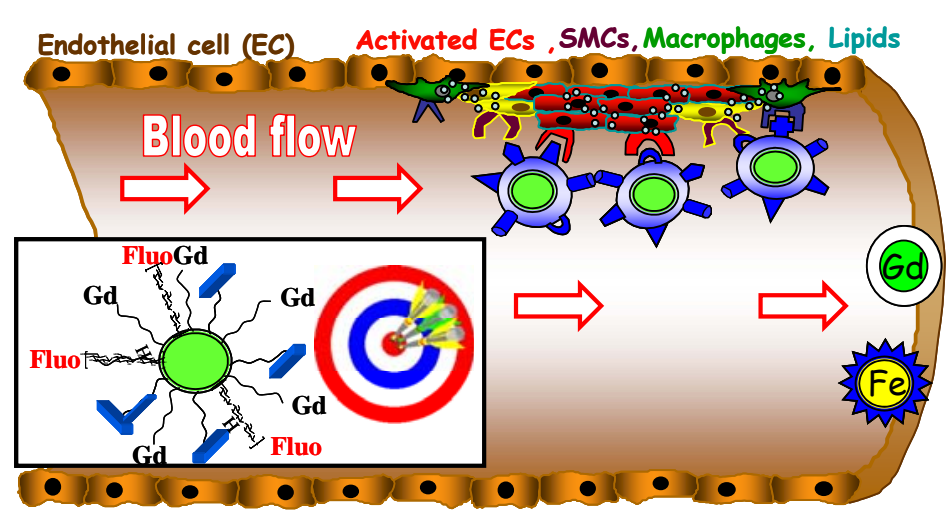

Figure 3: Targeting the vulnerable plaque with a bi-modal (optical-MRI) functionalized $M R$ contrast agent. The non specific MR marker, i.e. gadolinium (Gd) or iron based contrast agent (Fe) will remain in the blood stream, whereas the targeted agent (as represented in the box) will bind to a specific receptor on the inflamed vessel wall via a ligand-receptor like interaction (ligand or specific chemical functions in blue). The optimal design combines high specificity for the vulnerable plaque (the target) and a high sensitivity (interpretable diagnostic images at low dose). By adding a fluorochrome (Fluo), it could be detected precisely at a low concentration using microscopic fluorescence imaging. Last but not least, the challenge is to choose the optimal cellular or molecular target, e.g. activated endothelial cells (ECs), smooth muscle cells (SMCs), macrophages, lipids, enzymes,...

Among the important players in the pathogenesis of the plaque, inflammation and adhesion molecules are considered to trigger vulnerability. Adhesion molecules are expressed at the surface of various activated cells in vulnerable plaque and a soluble fraction is also present in the blood circulation of patients at risk of rupture \{Ballantyne, 2005; Koh, 2005\}. Thus, they are considered as good candidates for targeting. Several studies have developed MR markers to target specific cell adhesion molecules, such as selectins or vascular adhesion molecule VCAM1 Laurent, 2004; Nahrendorf, 2006; Tsourkas, 2005\}. We proposed to associate the high resolution MRI with the injection of a specific MR contrast agent, obtained by grafting "key functions of the ligand of P-Selectin", an adhesion molecule expressed at the surface of activated platelets and endothelial cells \{Kappelmayer, 2004; Blondin, 2000\}, on a paramagnetic macromolecular gadolinium agent (carboxy-methyl-dextran DOTA-Gd, P717, Guerbet, France) \{Chaabane, 2004\}. The arterial wall contrast enhancement was evaluated in vivo with the new macromolecular gadolinium agent functionalized to target inflammation in ApoE-/- mice (Figures 3 and 4). 


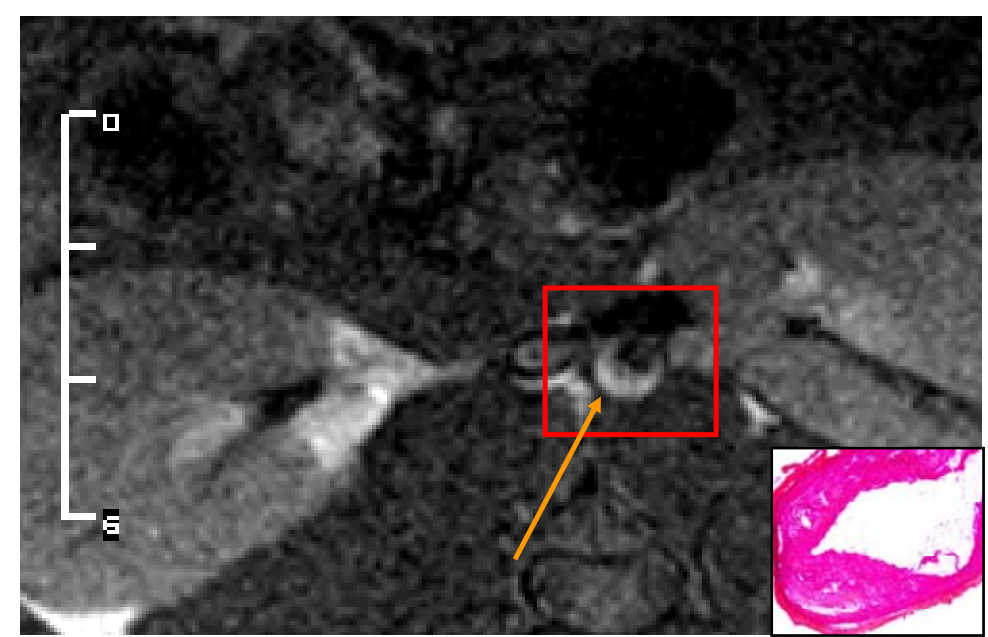

Figure 4: in vivo MRI with a bi-modal optical-MRI contrast agent, functionalized to target plaque inflammation. The transversal T1-weighted MR image (pixel size $=90 \times 90 \mu \mathrm{m} 2$, slice thickness $=1 \mathrm{~mm}$ ) of the abdominal aorta at the kidney level in this old ApoE-/- mouse, shows a strong, localized and persistent enhancement of the vessel wall (> $40 \%$ of signal increase, 82 minutes post intravenous injection, arrow), which was still visible 5 hours postinjection. The histology (box) confirms the presence of an important plaque at the same location \{Alsaid, 2006\}.

The biomimetic agent was synthesized by grafting key chemical functions for the interaction between P-selectin and its main ligand, PSGL-1, on the backbone of the carboxymethyl dextran gadolinium. A special care was taken to preserve the high relaxivity of the original blood pool agent, P717. We showed that this agent was able to be detected by a strong bright and persistent signal in the plaque, as seen in Figure 4. To improve the sensitivity and characterization, rhodamine was chemically added to enable fluorescent detection of the newly synthesized marker (Figure 5). The fluorescence of the compound allows a co-localization on microscopic preparation with inflammatory receptors on immunohistochemistry. The dual detection ensures to validate the in vivo results by the ex vivo preparation (Figure 5) with a co-localization and a complete characterization of lesions on histopathology. 


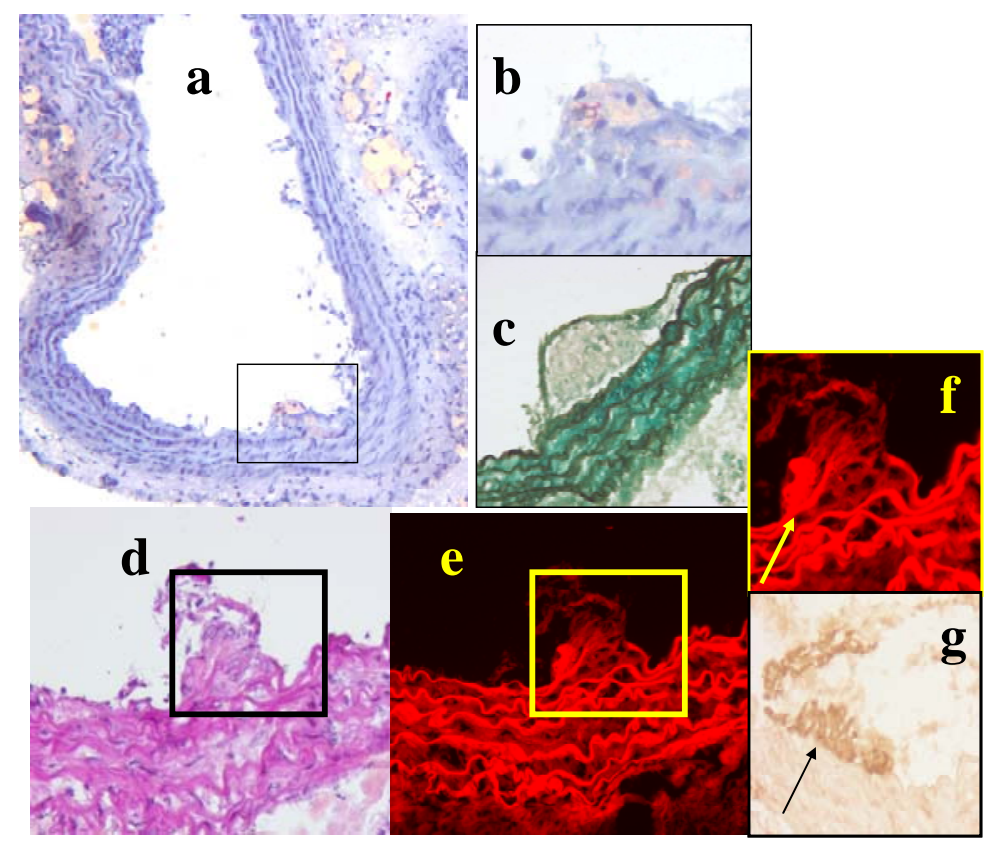

Figure 5: Ex vivo plaque characterization by histopathology and co-localization of the bimodal optical-MR marker with fluorescence microscopy in an early lesion of the aortic arch in an ApoE-/- mouse. Soudan III staining (a, low magnification, x10 and b, higher magnification x40) shows a small intimal lesion with lipid accumulation (in red). Trichrome of Masson (c) stains for elastic laminae (in green) and confirms the intimal proliferation. The lesion on standard hematoxylin eosin stain (d) co-localized with the presence of red fluorescence (rhodamine channel of fluorescence microscope) ( $e, x 20$ and $f, x 40)$ of the functionalized optical-MR marker (f, arrow) and co-localized with inflammation, signed by the presence of $P$ selectin on immunohistochemistry (g, arrow).

\section{Conclusion and perspectives}

The strategy of a marker for multimodalities is favoured by many groups as it can benefit from each technique \{Aikawa, 2007; Deguchi, 2006; Montet, 2006; Mulder, 2006; Sosnovik, 2005; Tsourkas, 2005; van Tilborg, 2006; Wickline, 2006; Winter, 2006\}. MRI brings high spatial and temporal resolution in vivo, even for deep structures such as the aortic vessel wall. Optical methods offer a greater sensitivity and may be very helpful for quantification of sparse epitopes. Moreover, it has been extensively used for in vitro tissue characterization using microscopic tools and for molecular bioengineering. Future works aim at developing nanotechnologies for both therapeutic and diagnostic for local delivery of drugs and in vivo monitoring by molecular imaging \{Lanza, 2002; Winter, 2006; McCarthy, 2006; Weissleder, 2006\}. However, for atherosclerosis and treatment of the vulnerable plaque, an important concern is the animal model, as there is no model for plaque rupture. In the ApoE-/- mouse, the most widely used model in molecular biology, acute cardiovascular events, i.e. myocardial infarction or stroke, are not observed and on histopathology, rupture have been reported only in 
the inominate artery (Figure 2) and is a rare phenomenon. Thus, design of dual imaging nanotechnologies for atherosclerosis is a challenging task and has to be performed in close collaboration between chemist, bioengineer, biologist, pathologist, imaging physicist and cardiovascular physician.

\section{References}

[1] Aikawa, E., M. Nahrendorf, et al. (2007). "Multimodality molecular imaging identifies proteolytic and osteogenic activities in early aortic valve disease." Circulation 115 (3): 377-86.

[2] Alsaid, H., G. De Souza, et al. (2006). Molecular Imaging of Inflammation in Atherosclerosis Plaque Using Functionalized MRI Contrast Agent . Proc. Intl. Soc. Mag. Reson. Med., 14, Seattle.

[3] Amirbekian, S., J. S. Aguinaldo, et al. (2006). Imaging of Atherosclerosis In Vivo Using a Magnetic Resonance Contrast Probe Molecularly Targeted to Matrix Metalloproteinases (MMPs). Proc. Intl. Soc. Mag. Reson. Med., 14, Seattle.

[4] Ballantyne, C. M. and V. Nambi (2005). "Markers of inflammation and their clinical significance." Atheroscler Suppl 6 (2): 21-9.

[5] Blondin, C., I. Bataille, et al. (2000). "Polysaccharides for vascular cell targeting." Crit Rev Ther Drug Carrier Syst 17 (4): 327-75.

[6] Chaabane, L., E. Canet, et al. (2000). "Microimaging of atherosclerotic plaque in animal models." Magn Reson Mater Phy 11 (1-2): 58-60.

[7] Chaabane, L., N. Pellet, et al. (2004). "Contrast enhancement in atherosclerosis development in a mouse model: in vivo results at 2 Tesla." Magn Reson Mater Phy 17 (3-6): 188-95.

[8] Chaabane, L., E. C. Soulas, et al. (2003). "High-resolution magnetic resonance imaging at 2 Tesla: potential for atherosclerotic lesions exploration in the apolipoprotein E knockout mouse." Invest Radiol 38 (8): 532-8.

[9] Coleman, R., T. Hayek, et al. (2006). "A mouse model for human atherosclerosis: long-term histopathological study of lesion development in the aortic arch of apolipoprotein E-deficient (E0) mice." Acta Histochem 108 (6): 415-24.

[10] Corot, C., P. Robert, et al. (2006). "Recent advances in iron oxide nanocrystal technology for medical imaging." Adv Drug Deliv Rev 58 (14): 1471-504.

[11] Deguchi, J. O., M. Aikawa, et al. (2006). "Inflammation in atherosclerosis: visualizing matrix metalloproteinase action in macrophages in vivo." Circulation 114 (1): 55-62.

[12] Gupta, A. K. and M. Gupta (2005). "Synthesis and surface engineering of iron oxide nanoparticles for biomedical applications." Biomaterials 26 (18): 3995-4021.

[13] Jaffer, F. A., P. Libby, et al. (2006). "Molecular and cellular imaging of atherosclerosis: emerging applications." J Am Coll Cardiol 47 (7): 1328-38.

[14] Kappelmayer, J., B. Nagy, Jr., et al. (2004). "The emerging value of P-selectin as a disease marker." Clin Chem Lab Med 42 (5): 475-86.

[15] Koh, K. K., S. H. Han, et al. (2005). "Inflammatory markers and the metabolic syndrome: insights from therapeutic interventions." J Am Coll Cardiol 46 (11): 1978-85. 
[16] Kooi, M. E., V. C. Cappendijk, et al. (2003). "Accumulation of ultrasmall superparamagnetic particles of iron oxide in human atherosclerotic plaques can be detected by in vivo magnetic resonance imaging." Circulation 107 (19): 2453-8.

[17] Lanza, G. M., X. Yu, et al. (2002). "Targeted antiproliferative drug delivery to vascular smooth muscle cells with a magnetic resonance imaging nanoparticle contrast agent: implications for rational therapy of restenosis." Circulation 106 (22): 2842-7.

[18] Laurent, S., L. Vander Elst, et al. (2004). "Synthesis and physicochemical characterization of GdDTPA-B(sLex)A, a new MRI contrast agent targeted to inflammation." Bioconjug Chem 15 (1): 99-103.

[19] Libby, P. (2002). "Inflammation in atherosclerosis." Nature 420 (6917): 868-74.

[20] McCarthy, J. R., F. A. Jaffer, et al. (2006). "A macrophage-targeted theranostic nanoparticle for biomedical applications." Small 2 (8-9): 983-7.

[21] McEver, R. P. and R. D. Cummings (1997). "Perspectives series: cell adhesion in vascular biology. Role of PSGL-1 binding to selectins in leukocyte recruitment." J Clin Invest 100 (3): 485-91.

[22] McFadden, E. P., L. Chaabane, et al. (2004). "In vivo magnetic resonance imaging of large spontaneous aortic aneurysms in old apolipoprotein E-deficient mice." Invest Radiol 39 (10): 58590.

[23] Montet, X., K. Montet-Abou, et al. (2006). "Nanoparticle imaging of integrins on tumor cells." Neoplasia 8 (3): 214-22.

[24] Mulder, W. J., G. J. Strijkers, et al. (2006). "Lipid-based nanoparticles for contrast-enhanced MRI and molecular imaging." NMR Biomed 19 (1): 142-64.

[25] Nahrendorf, M., F. A. Jaffer, et al. (2006). "Noninvasive vascular cell adhesion molecule-1 imaging identifies inflammatory activation of cells in atherosclerosis." Circulation 114 (14): 1504-11.

[26] Nighoghossian, N., L. Derex, et al. (2005). "The vulnerable carotid artery plaque: current imaging methods and new perspectives." Stroke 36 (12): 2764-72.

[27] Ruehm, S. G., C. Corot, et al. (2001). "Magnetic resonance imaging of atherosclerotic plaque with ultrasmall superparamagnetic particles of iron oxide in hyperlipidemic rabbits." Circulation 103 (3): $415-22$.

[28] Sosnovik, D. E., M. Nahrendorf, et al. (2007). "Molecular magnetic resonance imaging in cardiovascular medicine." Circulation 115 (15): 2076-86.

[29] Sosnovik, D. E., E. A. Schellenberger, et al. (2005). "Magnetic resonance imaging of cardiomyocyte apoptosis with a novel magneto-optical nanoparticle." Magn Reson Med 54 (3): 718-24.

[30] Sosnovik, D. E. and R. Weissleder (2007). "Emerging concepts in molecular MRI." Curr Opin Biotechnol 18 (1): 4-10.

[31] Stary, H. C., A. B. Chandler, et al. (1995). "A definition of advanced types of atherosclerotic lesions and a histological classification of atherosclerosis. A report from the Committee on Vascular Lesions of the Council on Arteriosclerosis, American Heart Association." Circulation 92 (5): 135574.

[32] Tsourkas, A., V. R. Shinde-Patil, et al. (2005). "In vivo imaging of activated endothelium using an anti-VCAM-1 magnetooptical probe." Bioconjug Chem 16 (3): 576-81. 
[33] van Tilborg, G. A., W. J. Mulder, et al. (2006). "Annexin A5-functionalized bimodal lipid-based contrast agents for the detection of apoptosis." Bioconjug Chem 17 (3): 741-9.

[34] Wasserman, B. A., W. I. Smith, et al. (2002). "Carotid artery atherosclerosis: in vivo morphologic characterization with gadolinium-enhanced double-oblique MR imaging initial results." Radiology 223 (2): 566-73.

[35] Weinreb, D. B., J. G. Aguinaldo, et al. (2007). "Non-invasive MRI of mouse models of atherosclerosis." NMR Biomed 20 (3): 256-64.

[36] Weissleder, R. (2006). "Molecular imaging in cancer." Science 312 (5777): 1168-71.

[37] Wickline, S. A., A. M. Neubauer, et al. (2006). "Applications of nanotechnology to atherosclerosis, thrombosis, and vascular biology." Arterioscler Thromb Vasc Biol 26 (3): 435-41.

[38] Winter, P. M., S. D. Caruthers, et al. (2003). "Improved molecular imaging contrast agent for detection of human thrombus." Magn Reson Med 50 (2): 411-6.

[39] Winter, P. M., A. M. Neubauer, et al. (2006). "Endothelial \{alpha\}\{nu\} \{beta\}3 Integrin-Targeted Fumagillin Nanoparticles Inhibit Angiogenesis in Atherosclerosis." Arterioscler Thromb Vasc Biol .

[40] Wu, J. T. and L. L. Wu (2006). "Linking inflammation and atherogenesis: Soluble markers identified for the detection of risk factors and for early risk assessment." Clin Chim Acta 366 (1-2): 74-80.

[41] Yuan, C., W. S. Kerwin, et al. (2002). "Contrast-enhanced high resolution MRI for atherosclerotic carotid artery tissue characterization." J Magn Reson Imaging 15 (1): 62-7.er. Science . 2006;312:1168-71. 